\title{
Medicina Ortomolecular Baseada em Evidência
}

\author{
Luís Beck da Silva N eto, Jorge Pinto Ribeiro
}

PortoAlegre, RS

A expectativa em torno dos antioxidantes e seus potenciais benefícios à saúde estende-se há décadas. Inicialmente, havia evidências observacionais de que pessoas que consumiam mais frutas e verduras apresentavam menores riscos de câncer e de doenças cardiovasculares ${ }^{1}$. Na busca de explicações para este fato, observou-se que substâncias contidas nas frutas e verduras poderiam diminuir a oxidação passiva de moléculas de DNA e, com isso, diminuir a probabilidade da transformação inapropriada das células ${ }^{2}$. Também as lipoproteínas de baixa densidade (LDL), quando submetidas a dano oxidativo, tornar-se-iam mais aterogênicas ${ }^{3}$. Posteriormente, o conhecimento evoluiu para o ponto de dispormos de grandes estudos observacionais que avaliaram a associação entre vitaminas antioxidantes e doença coronária ${ }^{4,5}$, sugerindo potencial benefício do emprego de altas doses de vitamina $E$, mas não de vitamina $C$.

É característica dos estudos observacionais a capacidade de estabelecer associações entre uma exposição e uma doença. Associações estas que, independentemente do grau de significância estatística, não podem estabelecer causalidade ${ }^{6}$. É preciso ficar clara a necessidade de ensaios clínicos para embasar condutas clínicas preventivas de saúde. O conceito atual de medicina baseada em evidência indica que as recomendações para os pacientes precisam estar provadas sobre o seu real benefício ${ }^{7,8}$. No caso dos suplementos vitamínicos, havia, até aqui, uma evidência epidemiológica e uma explicação plausível para o fato. No entanto, faltava qualquer comprovação da relação causa-efeito entre o uso de antioxidantes e doenças cardiovasculares.

Sem perder tempo, a indústria de produtos alimentícios despejou no mercado, inicialmente americano, toneladas de suplementos vitamínicos de todos os tipos com a mensagem de melhorar a saúde e diminuir riscos. Isto se deu com certa permissividade, em vista de serem as vitaminas submetidas à legislação de alimento e não estarem sujeitas ao rígido escrutínio reservado às novas drogas. Nos Estados Unidos da América, dezenas de milhões de dólares foram gastos com esta crença ${ }^{2}$. Em nosso país, um apreciável número de médicos tem recomendado altas doses de suplementos vitamínicos com objetivos de prevenção primária e secundária, estabelecendo na prática a chamada medicina orto-

Hospital de Clínicas de Porto Alegre - UFRGS

Correspondência: Jorge Pinto Ribeiro - Hospital de Clínicas de Porto Alegre Serv de Cardiologia - Rua Ramiro Barcelos, 2350 - 90035-007 - Porto Alegre, RS Recebido para publicação em 30/1/97

Aceito em 21/5/97 molecular ${ }^{9,10}$. Esta abordagem parecia atrativa, mas não dispunha, até o momento, de qualquer comprovação de eficácia baseada em ensaios clínicos. Entretanto, resultados recentemente publicados permitem um posicionamento contemporâneo para aqueles que optam por praticar a medicina baseada em evidência.

Até o momento, a evidência mais consistente que dispúnhamos sobre efeitos de antioxidantes era do ATBC Cancer Prevention Trial ${ }^{11}$. Este foi um ensaio-clínico randomizado, duplo-cego, com seguimento de 29.133 homens fumantes por cinco a oito anos, que demonstrou ausência de efeito protetor de vitamina E e beta-caroteno na incidência de câncer de pulmão e outros cânceres. Os resultados desse ensaio sugerem a hipótese de que estes suplementos poderiam, inclusive, ser deletérios. Outros estudos menores, como o Polyp Prevention ${ }^{12}$, ensaio-clínico que testou as vitaminas $\mathrm{C}$ e $\mathrm{E}$ na recorrência de adenomas de cólon e reto, reforçam estes resultados. Além disso, o potencial teratogênico da vitamina A foi sugerido por uma grande coorte ${ }^{13}$.

Entretanto, dois ensaios clínicos e um estudo de coorte recentemente publicados apresentam dados muito interessantes. O primeiro arrolou 22.071 pessoas (todos médicos) saudáveis, entre 40 e 84 anos e randomizou os indivíduos para receberem 50mg de beta-caroteno em dias alternados ou placebo. Após 12 anos de seguimento, foi constatado que a incidência de câncer de pulmão, de câncer em geral, de doenças cardiovasculares e, enfim, a mortalidade, foi idêntica nos dois grupos ${ }^{14}$. O segundo ensaio-clínico envolveu 18.314 fumantes, ex-fumantes e trabalhadores expostos ao asbesto, que foram randomicamente alocados para receber uma combinação de beta-caroteno $(30 \mathrm{mg}$ por dia) e vitamina A (25.000UI de retinol por dia) ou placebo, tendo como desfechos a incidência de câncer de pulmão e doenças cardiovasculares ${ }^{15}$. Este ensaio, além de não mostrar qualquer benefício no uso de beta-caroteno, demonstrou uma significativa associação positiva entre o uso de vitamina A e câncer de pulmão (risco relativo de 1,28, com intervalos de confiança de $95 \%$ de $1,04-1,57 ; \mathrm{p}=0,02)$. Portanto, vitamina A poderia aumentar o risco para câncer de pulmão, se tomada regularmente em suplementos vitamínicos, por pessoas sem déficit vitamínico, que sejam fumantes ou expostas ao asbesto. $\mathrm{O}$ estudo de coorte de mulheres pósmenopáusicas ${ }^{16}$, recentemente publicado, lança alguma luz no entendimento dos resultados negativos dos ensaios clínicos citados. Este estudo examinou a dieta (e não os suplementos vitamínicos), como preditiva do risco de morte 
cardiovascular, mostrando que as mulheres que ingerem mais vitaminas $\mathrm{A}, \mathrm{Ce} \mathrm{E}$ nas suas dietas têm menor risco de doença cardiovascular. Porém, o uso de suplementos vitamínicos não ofereceu o mesmo benefício. É possível que as vitaminas contidas nas frutas e verduras sejam meras marcadoras de benefício, mas não propriamente as responsáveis pela proteção. $O$ consumo de frutas, provavelmente por estar associado a um estilo de vida saudável e uma postura que reflete uma vontade de viver, pode trazer benefício a estas mulheres, mas isto não se deve, necessariamente, às vitaminas A ou E ou C.

Outras pesquisas serão necessárias para melhor elucidar estes fatos. Entretanto, mais uma vez, conclui-se que os suplementos vitamínicos carecem de propriedades protetoras. Há, no entanto, uma exceção: a administração de vitamina Ea pacientes com cardiopatia isquêmica documentada pode diminuir a incidência de infartos do miocárdio, ${ }^{17} \mathrm{O}$ que foi demonstrado pelo ensaio-clínico CHAOS, no qual 2.002 pacientes foram randomizados para receber $400 \mathrm{a}$ $800 \mathrm{mg}$ de vitamina E ou placebo por uma média de 510 dias.
Nesse ensaio houve uma redução de $47 \%$ (risco relativo de 0,53 ; com intervalos de confiança de $95 \%$ de $0,34-0,83$; $\mathrm{p}=0,005$ ) na incidência de infartos fatais e não fatais no grupo tratado em comparação ao grupo placebo. Este foi o primeiro ensaio-clínico randomizado que mostrou benefício de uma vitamina antioxidante neste contexto. Entretanto, é importante ressaltar que estes resultados não podem ser extrapolados para pacientes sem evidência de doença coronária, que, por consistirem grupo de menor risco, possivelmente obtenham menor benefício.

Portanto, a avaliação crítica da literatura científica disponível, na forma de ensaios clínicos randomizados, permite dizer que os cardiologistas já podem praticar o que chamaríamos de medicina ortomolecular baseada em evidência. Até que outros estudos sejam divulgados, a vitamina E pode ser prescrita para pacientes com doença aterosclerótica coronária estabelecida. Qualquer outro tipo de prescrição de suplementos vitamínicos, com objetivo de prevenção primária ou secundária, é desprovido de confirmação científica e, em algumas situações, poderá aumentar o risco.

\section{Referências}

1. Peto R, Doll R, Buckley JD, Sporn MB - Can dietary beta caroteno materially reduce human cancer rates? Nature 1981; 290: 201-8.

2. Greenberg ER, Sporn MB - Antioxidant vitamins, cancer, and cardiovascular disease. N Engl J Med 1996; 334: 1189-90.

3. Steinberg D, Pathasarathy S, Carew TE, Khoo JC, Witzum JL - Beyond cholesterol: modifications of low-density lipoproteins that increase its atherogenicity. N Engl J Med 1989; 320: 915-24.

4. RimmEB, Stampfer MJ, Ascherio A, Giovanucci E, Colditz GA, Willet WC-Vitamin E consumption and the risk of coronary heart disease in men. NEngl J Med 1993; 328: 1450-6.

5. Stampfer MJ, Hennekens CH, Manson JE, Colditz GA, Rosner B, Willet WC Vitamin E consumption and the risk of coronary heart disease in women. NEngl J Med 1993; 328: 1444-9.

6. Grisso JA - Making comparisons. Lancet 1993; 342: 157-60.

7. Evidence-Based Medicine Working Group - A new approach to teaching the practice of medicine. JAMA 1992; 268: 2420-5.

8. Guyatt GH, Sackett DL, Cook DJ, for The Evidence-Based Medicine Working Group. User's Guides to the Medical Literature. II - How to use an article about therapy or prevention. A. Are the results of the study valid? JAMA 1993; 270: 2598-601.

9. McCarty MF - Toward a "bio-energy supplement" - a prototype for functional orthomolecular supplementation. Med Hypotheses 1981; 7: 4.
10. Hauser SP - Unproven methods in cancer treatment. Curr Opin Oncol 1993; $5: 4$.

11. The Alpha-Tocopherol, Beta Carotene Cancer Prevention Group - The effect of vitamin $E$ and beta carotene on the incidence of lung cancer and other cancers in male smokers. N Engl J Med 1994; 330: 1029-35.

12. Polyp Prevention Study Group - A clinical trial of antioxidant vitamins to prevent colorectal adenoma. N Engl J Med 1994; 331: 141-7.

13. Rothman KJ, Moore LL, Singer MR, Nguyen UDT, Mannino S, Milunsky A - Teratogenicity of high vitamin A intake. N Engl J Med 1995; 333: 1369-73.

14. Hennekens $\mathrm{CH}$, Buring JE, Manson JE et al - Lack of effect of long term supplementation with beta corotene on the incidence of malignant neoplasms and cardiovascular diseases. N Engl J Med 1996; 334: 1145-9.

15. Omenn GS, Goodman GE, Thornquist MD et al - Effect of a combination of beta carotene and vitamin $\mathrm{A}$ on lung cancer and cardiovascular disease. $\mathrm{NEngl} \mathrm{J} \mathrm{Med}$ 1996; 334: 1150-5.

16. Kushi LH, Folsom AR, Prineas RJ et al - Dietary antioxidant vitamins and death from coronary heart disease in postmenopause women. N Engl J Med 1996; 334: $1156-62$.

17. Stephens NG, Parsons A, Schofield PMet al - Randomised controlled trial of vitamin E in patients with coronary disease: Cambridge Heart Antioxidant Study (CHAOS). Lancet 1996; 347: 781-5. 